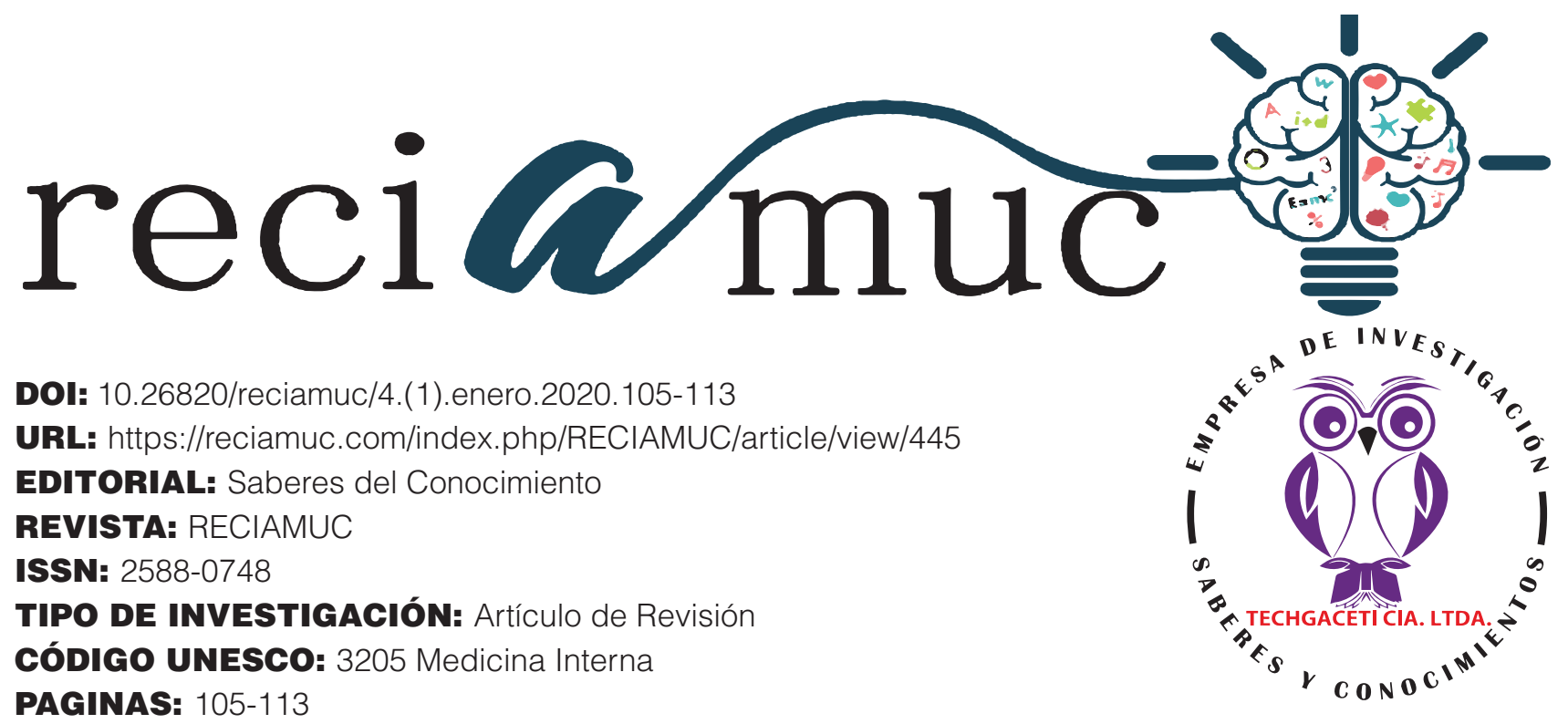

\title{
V.E.D.A terapéutica en hemorragia digestiva alta
} Therapeutic V.E.D.A in upper gastrointestinal bleeding

\section{V.E.D.A terapêutica no sangramento gastrointestinal superior}

Jessica Alexandra Ledesma Ledesma'; Marcia Julieth Rivera Mera2; Luis Javier Alvarez Moyon3; Karol Natalie Galarza Avila ${ }^{4}$

RECIBIDO: 18/11/2019 ACEPTADO: 20/12/2019 PUBLICADO: 31/01/2020

1. Médico; Investigador Independiente; Guayaquil, Ecuador; j.ldsm@hotmail.com; (D) https://orcid.org/00000002-3852-2811

2. Médico; Investigador Independiente; Guayaquil, Ecuador; july_rivme@hotmail.com; $\mathbb{D}$ https://orcid.org/00000001-6520-9890

3. Médico; Investigador Independiente; Guayaquil, Ecuador; lujam898989@gmail.com; (D) https://orcid.org/00000002-6042-0395

4. Médico; Investigador Independiente; Guayaquil, Ecuador; karito-naty@hotmail.com; D https://orcid.org/00000003-0458-3584

\section{CORRESPONDENCIA}

Jessica Alexandra Ledesma Ledesma

j.ldsm@hotmail.com

\section{Guayaquil, Ecuador}




\title{
RESUMEN
}

La hemorragia digestiva alta es aquella que se origina en una lesión situada entre el esfínter esofágico superior y el ángulo de Treitz. Este sangrado debe ser de suficiente magnitud como para producir hematemesis o melena, puede originarse del propio tubo digestivo o de estructuras adyacentes que vierten a él su contenido hemático, en el espacio comprendido entre los dos puntos referidos. Incluye desde cuadros clínicos manifestados por anemia crónica relacionadas con pérdidas microscópicas hasta formas graves que ponen en peligro la vida del paciente y que requieren una rápida resucitación, diagnóstico y tratamiento de la causa de la hemorragia. El proceso diagnóstico de la hemorragia digestiva alta, consta de una fase sindrómica sustentada en la historia clínica, la exploración física y las exploraciones complementarias básicas para identificar la lesión causal, que se alcanza fundamentalmente mediante la endoscopia digestiva alta. La endoscopia digestiva alta es una técnica que permite visualizar el interior de la porción alta del tubo digestivo (esófago, estómago y duodeno) de una forma directa mediante la introducción de un endoscopio flexible por la boca, obteniéndose una imagen en tiempo real. La evaluación endoscópica en un paciente con hemorragia digestiva alta, permite realizar un diagnóstico preciso en un alto porcentaje de los casos si se realiza tempranamente y permite pronosticar el riesgo que esta lesión tiene de repetir el sangrado, permitiendo así sugerir la terapia endoscópica a los pacientes con mayor riesgo. La metodología usada es descriptiva, con un enfoque documental, es decir, revisar fuentes disponibles en la red, como google académico, con contenido oportuno, actualizado y relevante desde el punto de vista científico que enriquezca el análisis del tema planteado en este artículo.

Palabras clave: Hematemesis, Hemorragia Digestiva Alta, Endoscopia, Tubo Digestivo, Melena, Inestabilidad Hemodinámica, Anemia.

\begin{abstract}
Upper gastrointestinal bleeding is one that originates from a lesion located between the upper esophageal sphincter and the Treitz angle. This bleeding must be of sufficient magnitude to produce hematemesis or mane, it can originate from the digestive tract itself or from adjacent structures that pour its blood content into it, in the space between the two points mentioned. It includes clinical pictures manifested by chronic anemia related to microscopic losses to serious forms that endanger the patient's life and that require rapid resuscitation, diagnosis and treatment of the cause of bleeding. The diagnostic process of upper gastrointestinal bleeding consists of a syndromic phase based on the clinical history, physical examination and basic complementary examinations to identify the causal lesion, which is mainly achieved through upper digestive endoscopy. The upper digestive endoscopy is a technique that allows visualizing the inside of the upper portion of the digestive tract (esophagus, stomach and duodenum) in a direct way by introducing a flexible endoscope through the mouth, obtaining a real-time image. Endoscopic evaluation in a patient with high gastrointestinal bleeding, allows an accurate diagnosis in a high percentage of cases if done early and allows to predict the risk that this injury has to repeat bleeding, thus allowing patients to suggest endoscopic therapy with greater risk. The methodology used is descriptive, with a documentary approach, that is, to review sources available on the web, such as google scholar, with timely, updated and scientifically relevant content that enriches the analysis of the topic raised in this article.
\end{abstract}

Keywords: Hematemesis, Upper Digestive Hemorrhage, Endoscopy, Digestive Tube, Mane, Hemodynamic Instability, Anemia.

\section{RESUMO}

O sangramento gastrointestinal superior é aquele que se origina de uma lesão localizada entre o esfíncter superior do esôfago e o ângulo de Treitz. Esse sangramento deve ser de magnitude suficiente para produzir hematêmese ou crina; pode originar-se do próprio trato digestivo ou de estruturas adjacentes que despejam seu conteúdo sanguíneo nele, no espaço entre os dois pontos mencionados. Inclui quadros clínicos manifestados por anemia crônica relacionada a perdas microscópicas para formas graves que colocam em risco a vida do paciente e requerem rápida ressuscitação, diagnóstico e tratamento da causa do sangramento. O processo diagnóstico de sangramento gastrointestinal superior consiste em uma fase sindrômica baseada na história clínica, no exame físico e em exames complementares básicos para identificar a lesão causal, que é alcançada principalmente por endoscopia digestiva alta. A endoscopia digestiva alta é uma técnica que permite visualizar o interior da porção superior do trato digestivo (esôfago, estômago e duodeno) de maneira direta, introduzindo um endoscópio flexível pela boca, obtendo uma imagem em tempo real. A avaliação endoscópica em um paciente com sangramento gastrointestinal alto, permite um diagnóstico preciso em uma alta porcentagem de casos, se feita precocemente e permite prever o risco de que essa lesão tenha que repetir o sangramento, permitindo que os pacientes sugiram terapia endoscópica com maior risco. A metodologia utilizada é descritiva, com abordagem documental, ou seja, para revisar fontes disponíveis na web, como o google scholar, com conteúdo oportuno, atualizado e cientificamente relevante que enriquece a análise do tópico levantado neste artigo.

Palavras-chave: Hematêmese, Hemorragia Digestiva Superior, Endoscopia, Tubo Digestivo, Juba, Instabilidade Hemodinâmica, Anemia. 


\section{Introducción}

La hemorragia digestiva alta constituye un motivo frecuente de consulta. Consiste en la pérdida de sangre hacia la luz del tubo digestivo en una zona comprendida entre el esfínter esofágico superior y el ángulo de Treitz. Este sangrado puede proceder de órganos digestivos o de estructuras adyacentes que vierten su contenido en este espacio, por conductos naturales (árbol biliopancreático) o patológicos (fístulas espontáneas, traumáticas o quirúrgicas).

Se manifiesta clínicamente por hematemesis y melena, independientemente de la lesión de origen, diferenciándose sólo por su magnitud, evidenciable a través de la presencia o ausencia de compromiso hemodinámico y magnitud del mismo. Al inicio, toda hemorragia digestiva alta debe considerarse como potencialmente grave, y por lo tanto, merecedora de esfuerzos diagnósticos y terapéuticos intensos. La morbilidad y mortalidad del paciente se relaciona con ciertos factores de riesgos, como la edad, enfermedades asociadas magnitud de la hemorragia, origen de la misma, alcoholismo, ingesta de medicamentos, etc.

La endoscopía con intención terapéutica se considera como la intervención más temprana a realizar para el control del sangrado y la prevención del re sangrado. Los predictores del pre sangrado son: edad avanzada, shock, inestabilidad hemodinámica, comorbilidades (Ios pacientes cirróticos que sobreviven a un episodio de sangrado tienen un riesgo de re sangrado que llega a 60 \% en 1 año (Machuca-Gómez, Abril 2008). Cada episodio de re sangrado está asociado a una mortalidad de $20 \%$.

Lo expuesto hasta ahora justifica claramente la importancia de profundizar en este tema, a lo largo de este artículo se realizó una revisión de la bibliografía especializada en el tema con el objetivo de exponer lo relativo a su diagnóstico, manejo y técnicas de tratamiento idónea que mejoren la respuesta médica y tiempo de malestar al paciente, disminuyendo las operaciones innecesarias.

\section{Metodología}

Esta investigación está dirigida al estudio "V.E.D.A terapéutica en hemorragia digestiva alta". Para realizarlo se usó una metodología descriptiva, con un enfoque documental, es decir, revisar fuentes disponibles en la red, como google académico, con contenido oportuno y relevante desde el punto de vista científico para dar respuesta a lo tratado en el presente artículo y que sirvan de inspiración para realizar otros proyectos. Las fuentes consultadas pueden ser estudiadas al final, en la bibliografía.

\section{Resultados}

La hemorragia digestiva alta es aquella que se origina en una lesión situada entre el esfínter esofágico superior y el ángulo de Treitz (formado por la 4ta porción duodenal y el yeyuno). Este sangrado debe ser de suficiente magnitud como para producir hematemesis o melena, puede proceder del propio tubo digestivo o de estructuras adyacentes que vierten a él su contenido hemáti$\mathrm{co}$, en el espacio comprendido entre los dos puntos referidos (por ejemplo por una fístula aorto-digestiva). Incluye desde cuadros clínicos manifestados por anemia crónica relacionadas con pérdidas microscópicas hasta formas graves que ponen en peligro la vida del paciente y que requieren una rápida resucitación, diagnóstico y tratamiento de la causa de la hemorragia (Montero, 2005).

Las causas más frecuentes son: lesiones mucosas agudas gastroduodenales, las úlceras pépticas duodenales y gástricas y los procesos relacionados con la hipertensión portal, que incluyen várices esofágicas y del techo gástrico, y la gastropatía hipertensiva. Otras causas menos frecuentes son el síndrome de Mallory-Weiss, el cáncer y leiomioma gástricos y las ectasias vasculares (Samaniego-Casco, 2005). La siguiente figura muestra el algoritmo de diagnóstico sugerido en hemorragia digestiva alta. 
Tabla 1. Algoritmo Diagnostico de Hemorragia Digestiva Alta

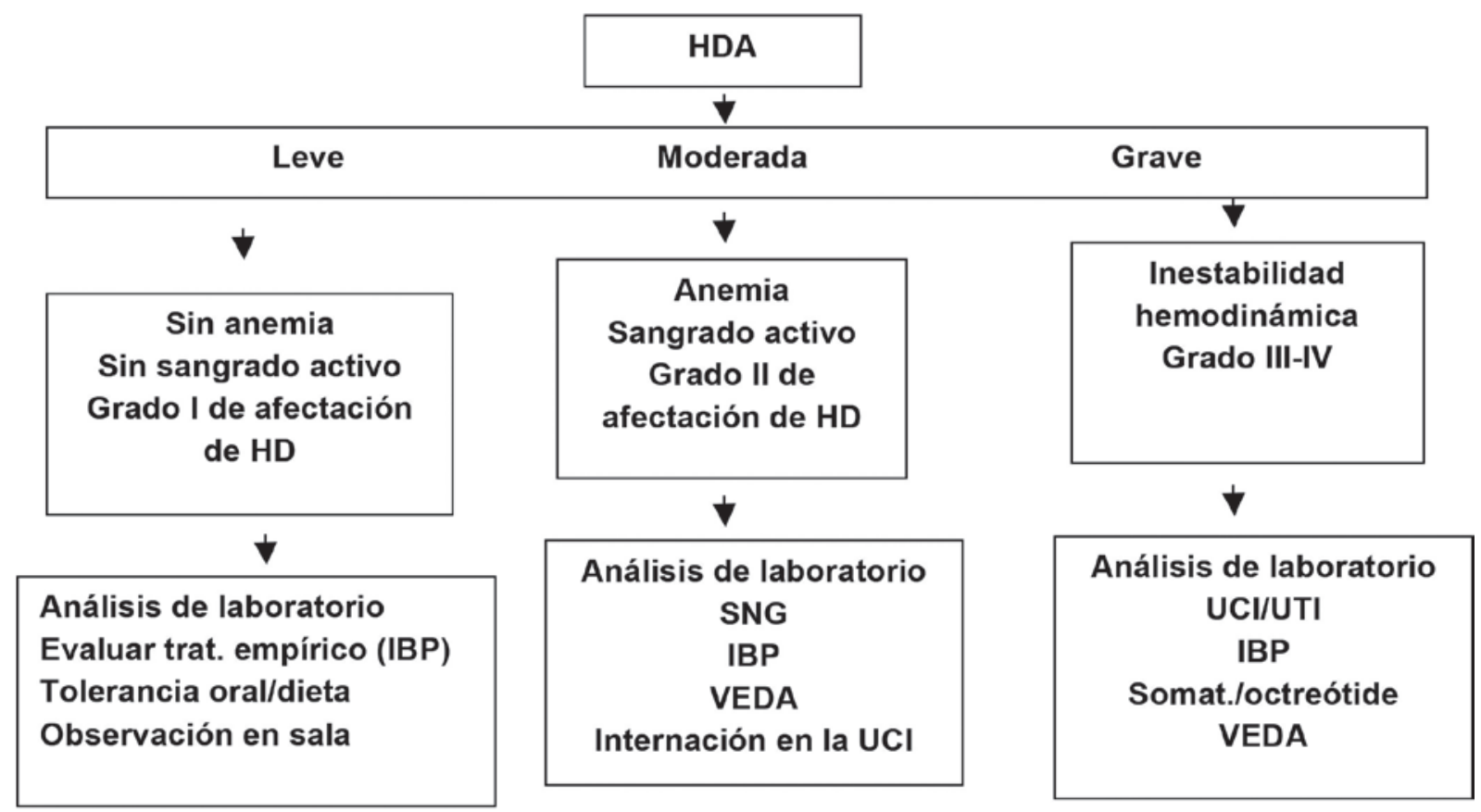

HDA: hemorragia digestiva alta; HD: hemorragia digestiva; IBP: inhibidores de la bomba de protones; SNG: sonda nasogástrica; VEDA: videoendoscopía digestiva alta; UCI: Unidad de Cuidados Intensivos; UTI: Unidad de Terapia Intensiva.

Fuente: (Riga-Villarruel, 2016)

El proceso diagnóstico de la hemorragia digestiva alta, como el de cualquier otra patología, consta de una fase sindrómica sustentada en la historia clínica, la exploración física y las exploraciones complementarias básicas (fundamentalmente el hemograma y la bioquímica sanguínea) y de otra etiológica, para identificar la lesión causal, que se alcanza fundamentalmente mediante la endoscopia digestiva alta.

Suele manifestar por hematemesis y/o melenas, dependiendo su forma de presentación del débito de la hemorragia y de la localización de la lesión sangrante. La presencia de sangre en el vómito se denomina hematemesis. Esta puede adoptar una coloración negruzca o rojo brillante (sangre fresca), dependiendo de que haya sido alterada o no por los fluidos gástricos. La deposición de heces muy oscuras, alquitranadas, brillantes, pegajosas, fétidas y de consistencia pastosa recibe el nombre de melena. La hematoquecia es la emisión de sangre roja por el ano, sola o mezclada con la deposición, y suele ser indicativa de hemorragia digestiva baja aunque en ocasiones es una forma de presentación de la hemorragia digestiva alta (HDA).

El examen físico permite confirmar la presencia de melenas mediante el tacto rectal, valorar la intensidad de la hemorragia y su repercusión hemodinámica. Es fundamental repetir la exploración física cada cierto tiempo debido a que el estado del paciente puede cambiar rápidamente. Puede observarse palidez de piel y mucosas, expresión de la pérdida hemática o del cortejo vegetativo acompañante; estigmas de hepatopatía crónica (arañas vasculares, eritema palmar, circulación venosa colateral, ascitis, hepatoesplenomegalia); la presencia de equimosis y/o petequias, que orienten a una enfermedad hematológica de base; la existencia de lesiones dérmicas (Ichiyanagu, 2006).

La medición de la frecuencia cardíaca y 
de la presión arterial en reposo aporta una inestimable información sobre la gravedad del sangrado. Los datos que la exploración física permite al clínico realizar una clasificación de la hemorragia digestiva abdominal en:

- HDA leve: presión arterial sistólica > 100 $\mathrm{mm} \mathrm{Hg}$ y frecuencia cardíaca < 100 lpm.

- HDA grave: Presión arterial sistólica $<100 \mathrm{~mm} \mathrm{Hg}$ y frecuencia cardíaca > 100 lpm, con cambios significativos con ortostatismo y evidencia de signos de hipo perfusión periférica (palidez, sudoración, frialdad de piel, pérdida de recuperación capilar, cianosis, livideces, alteraciones del nivel de conciencia).

En cuanto a la evaluación por exámenes de laboratorio el cociente urea/creatinina se ha considerado un parámetro de gran poder discriminativo entre el origen alto o bajo de la hemorragia gastrointestinal, para cifras mayores de 90-100 son capaces de diferenciar correctamente la hemorragia digestiva alta de la hemorragia digestiva baja en el 90\% de los casos (García-García, Martín-Lorenzoa, Torralba-Martíneza, \& Lirón-Ruiza, 2015). El estudio de la coagulación se hace imprescindible en todo proceso hemorrágico para detectar la presencia de una coagulo patía primaria o secundaria a una hepatopatía crónica subyacente.

Es conveniente comprobar la actividad de protrombina o tiempo de Quick, el tiempo de tromboplastina parcial y el recuento plaquetario. Se considera que existe riesgo de sangrado con cifras por debajo de 40.000 plaquetas $/ \mathrm{mm}$. Otro examen que aporta ayuda en el diagnóstico es el recuento leu- cocitario, se ha observado la existencia de leucocitosis (> 15000/mm3) que aparece pasadas entre 2 y 5 horas del inicio del sangrado (Montero, 2005).

La endoscopia inicial debe realizarse tan pronto como sea posible. En pacientes con hemorragia grave se debe realizar cuando se consiga su estabilidad hemodinámica. Si ésta no es posible y persiste la situación de shock, debe valorarse la indicación de cirugía urgente realizando una endoscopia peroperatoria. En el resto de pacientes, la endoscopia debería realizarse en las primeras 12 horas siguientes a su consulta en urgencias, idealmente dentro de las 6 primeras horas, y nunca por encima de las 24 horas ya que entonces disminuye de forma drástica su rendimiento diagnóstico. La terapia endoscópica es el tratamiento de primera elección en pacientes con úlceras con hemorragia activa, vaso visible no sangrante y coágulo adherido sobre la lesión.

La endoscopia digestiva alta es una técnica que permite visualizar el interior de la porción alta del tubo digestivo (esófago, estómago y duodeno) de una forma directa mediante la introducción de un endoscopio flexible por la boca, obteniéndose una imagen en tiempo real que tras un procesamiento electrónico es trasladada a un monitor donde es valorada por el especialista que maneja el endoscopio. La siguiente figura ilustra la visión del VEDA. 


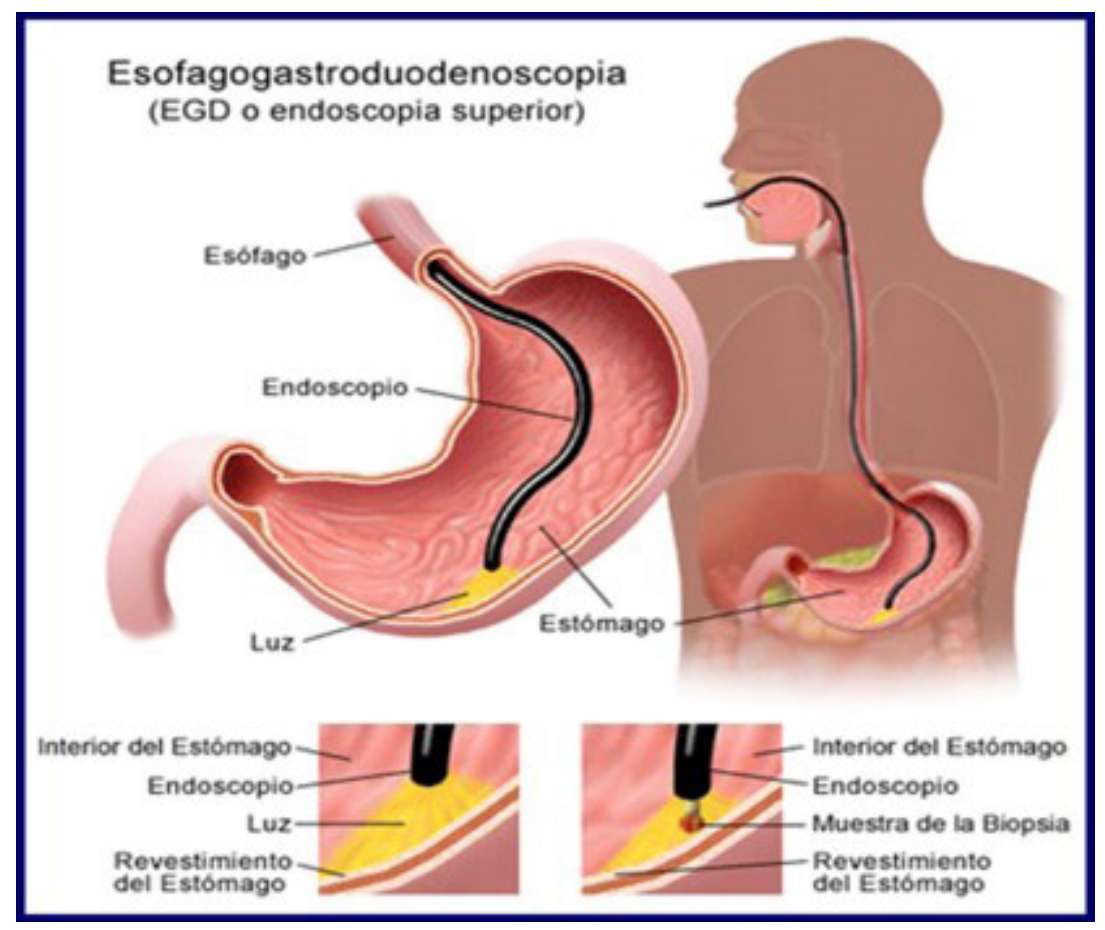

Figura 1. ¿Qué ocurre durante un video endoscopia digestiva alta?

Fuente: (Diario Salud, 2016)

La VEDA generalmente es tolerada correctamente por el paciente, se coloca un anestésico local en forma de spray o gel en la boca minutos previos al procedimiento. La endoscopia digestiva no sirve solo para ver y filmar el interior del esófago, estómago y duodeno, también puede ser usada para realizar biopsias y tratamiento de algunos problemas, como úlceras o várices sangrantes. A través del endoscopio es posible introducir una serie de herramientas, como pinzas de biopsia, lazos, agujas, sondas para escleroterapia o electrocauterio, balón de dilatación, redes y cestas (Muñoz-Navas, 2016).

La evaluación endoscópica en un paciente con hemorragia digestiva alta, no sólo permite realizar un diagnóstico preciso en un alto porcentaje de los casos si se realiza tempranamente, sino que además, la observación de ciertas características de las lesiones sangrantes permite en forma concomitante pronosticar el riesgo que esta lesión tiene de repetir el sangrado, permitiendo así sugerir la terapia endoscópica a los pacientes con mayor riesgo. Forrest descri- bió una clasificación que ha sido utilizada basándose en las diferentes características endoscópicas de úlceras pépticas gastroduodenales con sangrado activo o reciente, las cuales se asocian a diferentes riesgos de sangrado recurrente o persistente (Rodriguez-Torres, 2013).

El primer grupo, Forrest I, incluye a las úlceras pépticas con sangrado activo, pulsátil (Forrest IA) o sangrado en napa desde el lecho ulceroso (Forrest IB). El segundo grupo, Forrest II, incluye a úlceras sin sangrado activo al momento de su observación, pero con signos o estigmas de sangrado reciente en el lecho ulceroso, como el vaso visible (Forrest IIA) que se describe como una protuberancia pigmentada de superficie lisa, de menos de $3 \mathrm{~mm}$, la cual corresponde a un pequeño coágulo ubicado sobre el defecto de la pared del vaso que originó el sangrado y que proporciona una hemostasia inestable, el coágulo adherido (Forrest IIB) definido como una lesión amorfa, roja, café o negra, de tamaño variable, en general mayor a $5 \mathrm{~mm}$, adherida al lecho ulceroso y que no se desprende con aspiración o 
lavado del coágulo, señal de una hemostasia algo más avanzada (Vanina Lepore, Vanesa Sampo, \& Paola Storni, 2006).

La mácula plana (Forrest IIC) la cual se describe como una mancha pigmentada plana, sin relieve evidente en el lecho de la úlcera. Las lesiones tipo Forrest III corresponden exclusivamente a las úlceras pépticas con fondo limpio fibrinoso, sin ningún tipo de protuberancia o cambio en coloración (Vanina Lepore, Vanesa Sampo, \& Paola Storni, 2006). Según la clasificación de Forrest, la mayoría de los pacientes presentaban úlceras Forrest III y en segundo lugar úlceras Forrest II; esto revela que en la mayoría de los casos el sangrado es auto limitado, sin embargo debe tenerse en cuenta que éstos son pacientes internados en sala común en la mayoría de los casos, remitidos desde el servicio de urgencias, una vez que se encuentra superado el cuadro agudo de hemorragia digestiva alta.

Dentro de las ventajas de la aplicación de la endoscopía como técnica terapéutica podemos mencionar (Machuca-Gómez, Abril 2008):

- Presenta una alta sensibilidad y especificidad 92 a 95\%, como método diagnóstico.

- Permite identificar las causas de hemorragia digestiva alta, es una complicación presente entre el 15 al 20\% de los enfermos con úlcera péptica, existiendo un $80 \%$ con antecedentes de enfermedades ulcerosas.

- Identifica hemorragia varicosa, así como también hemorragia digestiva no varicosa; expansiones de las indicaciones endoscópicas en pacientes con adenocarcinomas gástricos, a pesar de que en ellos la cirugía es el tratamiento de elección.

- En la prevención de recidivas secundarias, la asociación de fármacos y técnicas endoscópicas es claramente superior que la utilización aislada de cualquiera de ellos; el tratamiento endoscópico comparado con drogas o placebo demostró disminuir el número de re sangrado, el número de cirugías y la mortalidad, en los pacientes con hemorragias digestivas alta no varicosa.

- En el caso de re sangrado se recomienda una segunda endoscopía y tratamiento endoscópico. La cirugía se reserva para el fracaso del segundo tratamiento endoscópico.

Dentro de las posibles complicaciones relevantes en la realización de la endoscopia digestiva alta se muestran:

- Derivadas de la sedación y anestésicos tópicos: los eventos adversos relacionados con la sedación intravenosa incluyen reacciones alérgicas, interacciones farmacológicas, depresión respiratoria, paro respiratorio e hipotensión.

- Complicaciones cardiovasculares: aunque inusuales, han sido descritas e incluyen dolor torácico, infarto de miocardio, paro cardiaco, hipotensión y arritmias dentro de las primeras 24 horas del procedimiento.

- Infecciones: pueden provenir de dos fuentes: la transmisión de microorganismos de paciente a paciente a través del material contaminado y otra, la transmisión de microorganismos desde el tracto gastrointestinal del paciente por medio del torrente sanguíneo a otros órganos o dispositivos protésicos implantados.

- Perforaciones: Cuando se produce una perforación, está suele afectar al tercio distal del esófago, aunque existen casos descritos de perforación en puntos anatómicos como el seno piriforme o divertículo de Zenker. El riesgo de perforación se eleva durante el manejo de tejidos anormales, siendo por tanto más probable durante la intervención con tejidos neoplásicos. Los síntomas de perfora-

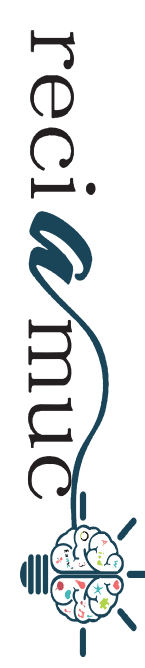


ción durante la endoscopia alta incluyen dolor severo y/o persistente (habitualmente localizado en región media-inferior del tórax), taquipnea, taquicardia, leucocitosis y fiebre. En otras ocasiones, la exploración física pondrá de manifiesto crepitación de partes blandas en cuello y tórax.

- Aspiración: para reducir el riesgo de aspiración se debe evitar el uso de anestésicos tópicos (para mantener el reflejo de deglución), prevenir la sobre sedación, mantener la cabecera de la cama con un ángulo de 30 - 45 grados, insuflar la menor cantidad de aire posible y aspirar a conciencia el contenido gástrico. Es fundamental el ayuno previo de al menos dos horas para líquidos y seis horas para sólidos. Una cuidadosa atención a los factores que predisponen a la aspiración bronquial, incluyendo la retención de contenido en el tubo digestivo por una obstrucción parcial de intestino delgado o la hemorragia masiva es crucial y se pueden presentar luego de una toma de biopsia.

- Hemorragia: es poco probable que suceda durante el procedimiento, cuando ocurre suele deberse al traumatismo producido por el paso del endoscopio.

- Incarceración del endoscopio: puede ocurrir si el paciente eructa durante la maniobra de retroflexión para examinar el fundus provocando el ascenso en "U" del endoscopio al esófago incarcerándose. Tras intentar enderezarlo y ante la resistencia del cardias que impedía el avance anterógrado, se introdujo un segundo endoscopio de menor calibre por la boca, el cual utilizaron para ejercer presión axial a nivel de la región curva del endoscopio consiguiendo así evaluar el estómago (Castillo-Vega, Noviembre 2008).

\section{Conclusiones}

El video endoscopía digestiva alta (VEDA) es el método de elección para evaluar el origen de la hemorragia digestiva alta. Lo ideal es realizarla en las primeras 24 horas del inicio del evento, en las que se identifica el origen con mayor certeza que si se realiza 72 horas después. La VEDA se ha convertido en el "tratamiento de oro" para diagnóstico de patología digestiva alta, ya que permite localizar e identificar el origen de la hemorragia, evaluar la velocidad de sangrado, definir cuál de todas la lesiones es la causa de sangrado y riesgo de re sangrado. El tratamiento endoscópico de las úlceras pépticas ha reducido el riesgo de recurrencia y la necesidad de cirugía. Esto se traduce en una estadía hospitalaria más corta, disminución en los costos y en el número de transfusiones sanguíneas, y menor morbilidad.

Luego de considerar las diversas ventajas y desventajas de la endoscopía digestiva alta, se concluye que la misma es una opción válida, que ha ampliado de manera considerable el diagnostico, pronóstico y tratamiento en la hemorragia digestiva alta. Debe ser considerada como una intervención primaria y temprana para establecer la causa del sangrado, y a partir de ello realizar el tratamiento y estimar el riesgo individual para la recurrencia de la hemorragia.

Se hace evidente la relevancia que tiene la hemorragia digestiva alta dentro de las afecciones más frecuentes, por lo cual se hace necesario profundizar en los conocimientos relacionados con el diagnóstico, atención y tratamiento, traduciendo esto en una mayor rapidez de los mismos (especialmente, en la pronta realización de la VEDA), lo cual redundaría en un mayor bienestar de los pacientes que lo padecen.

La endoscopía digestiva alta temprana cambia el curso natural del sangrado recurrente en pacientes de alto riesgo, reduciendo con ello la mortalidad, tratando complicaciones potencialmente letales. Una de las posibles complicaciones es la perforación o el desgarro de la pared del estómago o el 
esófago, que pueden requerir cirugía. En el sitio de la biopsia puede producirse un sangrado menor, el sangrado puede detenerse espontáneamente o controlarse por medio del endoscopio. Algunos pacientes pueden presentar una reacción a los sedantes o bien experimentar complicaciones por enfermedades cardíacas o pulmonares.

\section{Bibliografía}

Castillo-Vega. (Noviembre 2008). PROTOCOLOS Y DIRECTRICES EN ENDOSCOPIA. Fundación Española de Endoscópia Digestiva, 11. Obtenido de https://www.sap.org.ar/uploads/consensos/ consensos_consenso-nacional-de-urgencias-endoscopicas-en-pediatria-2016-comite-nacional-de-gastroenterologia-64.pdf

Diario Salud. (2016). Lo que necesitás saber de las endoscopías: riesgos y beneficios. Obtenido de Diario Salud: http://www.diariosalud.com.ar

García-García, M., Martín-Lorenzoa, J., Torralba-Martíneza, J., \& Lirón-Ruiza, R. (2015). Endoscopia urgente por hemorragia digestiva tras cirugía bariátrica. Algoritmo terapéutico. Cirugía Española, 93(2), 97-104. doi:10.1016/j.ciresp.2014.05.002

Ichiyanagu, C. (2006). Epidemiología de la Hemorragia Digestiva. Acta Médica Peruana, 23(3), 153-155. Obtenido de http://www.scielo.org.pe/scielo. php?script=sci_arttext\&pi$d=S 1728-59172006000300005$
Machuca-Gómez. (Abril 2008). LA ENDOSCOPIA EN LA HEMORRAGIA DIGESTIVA ALTA. . Revista de Posgrado de la VI Cátedra de Medicina. , 24-26.

Montero, F. (2005). Claves diagnósticas y terapéuticas para el manejo de la hemorragia digestiva alta en Urgencias. Emergencias, 17(1), 40 - 49. Obtenido de http://www.dep4.san.gva.es/contenidos/urg/archivos/guias/2005/HDA\%20(Emergencias\%202005).pdf

Muñoz-Navas. (2016). Protocolo de indicaciones, contraindicaciones y complicaciones de la endoscopia en el tracto digestivo superior. Medicine - Programa de Formación Médica Continuada Acreditado, 12(3), 152 - 156. doi:10.1016/j. med.2016.02.007

Riga-Villarruel. (2016). Guía de diagnóstico y tratamiento de hemorragia digestiva en pediatría. Consenso Nacional de Urgencias Endoscópicas en Pediatría 2016, 1 - 26.

Rodriguez-Torres. (2013). Causas de hemorragia digestiva alta no varicosa. Cirugía Paraguaya, 31(1), 15-18.

Samaniego-Casco. (2005). Hemorragia Digestiva Alta No Varicosa. Anales de la Facultad de Ciencias Médicas de la UNA, 38(4), 46-50.

Vanina Lepore, G., Vanesa Sampo, C., \& Paola Storni, M. (2006). LESIONES ENDOSCOPICAS MAS FRECUENTES EN UN GRUPO DE PACIENTES CON CLINICA DE HEMORRAGIA DIGESTIVA ALTA. Revista de Posgrado de la Vla Cátedra de Medicina, 158(1), 5 - 8. Obtenido de https://med.unne.edu. ar/revistas/revista158/2_158.htm

\section{CITAR ESTE ARTICULO:}

Ledesma Ledesma , J., Rivera Mera, M., Alvarez Moyon, L., \& Galarza Avila, K. (2020). V.E.D.A terapéutica en hemorragia digestiva alta. RECIMAUC, 105113. doi:10.26820/reciamuc/4.(1).enero.2020.105-113

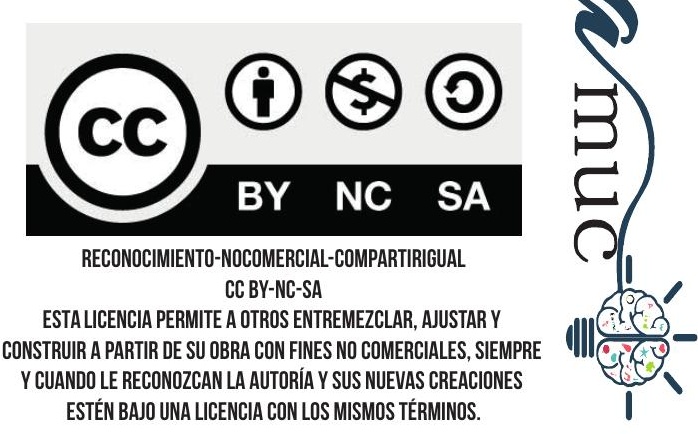

